\title{
microRNAs in cardiac regeneration and cardiovascular disease
}

\author{
WU GengZe ${ }^{1,2}$, HUANG Zhan-Peng ${ }^{1 *} \&$ WANG Da-Zhi ${ }^{1,3 *}$ \\ ${ }^{1}$ Department of Cardiology, Boston Children's Hospital, Harvard Medical School, Boston, MA 02115, USA; \\ ${ }^{2}$ Department of Cardiology, Chongqing Institute of Cardiology, Daping Hospital, Third Military Medical University, Chongqing 400042, \\ China; \\ ${ }^{3}$ Harvard Stem Cell Institute, Harvard University, Cambridge, MA 02138, USA
}

Received June 23, 2013; accepted July 18, 2013; published online August 21, 2013

\begin{abstract}
microRNAs (miRNAs) are a class of small non-coding RNAs, which have been shown important to a wide range of biological process by post-transcriptionally regulating the expression of protein-coding genes. miRNAs have been demonstrated essential to normal cardiac development and function. Recently, numerous studies indicate miRNAs are involved in cardiac regeneration and cardiac disease, including cardiac hypertrophy, myocardial infarction and cardiac arrhythmia. These observations suggest miRNAs play important roles in cardiology. In this review, we summarize the recent progress of studying miRNAs in cardiac regeneration and cardiac disease. We also discuss the diagnostic and therapeutic potential of miRNAs in heart disease.
\end{abstract}

microRNA, cardiac regeneration, cardiac remodeling, cardiovascular disease

Citation: Wu G Z, Huang Z P, Wang D Z. microRNAs in cardiac regeneration and cardiovascular disease. Sci China Life Sci, 2013, 56: 907-913, doi: $10.1007 / \mathrm{s} 11427-013-4534-9$

Historically, we have been focusing on protein-coding genes over the past decades and proteins have often been viewed as the final gene products that mediate most of the biological function in a given cell. However, more and more recent studies indicated that non-coding RNAs (ncRNAs) participate in the regulation of the expression of protein-coding genes therefore play essential roles in many biological processes. These findings uncovered that noncoding RNAs orchestrate a hidden layer of gene regulation network [1,2]. microRNAs (miRNAs), a class of small non-coding RNAs ( 20 nucleotides) [3,4], were first discovered in $C$. elegans two decades ago $[5,6]$. Now we know that miRNAs are present in virtually all plants [7] and animals [8-10]. Many miRNAs are evolutionary conserved, indicating they may serve as an ancient component of genetic regulation [11-14]. miRNAs are transcribed by RNA

*Corresponding author (email: zhuang@enders.tch.harvard.edu; dwang@enders.tch.harvard.edu) polymerase II (RNA-pol II) as primary transcripts (primiRNAs) [15], which contain stem-loop hairpin structure, and then subsequently processed by RNase III enzymes Drosha and Dicer [16-19]. The processed products are $\sim 22$ nt long miRNA duplexes with 2 nt overhangs on the $3^{\prime}$ end [20,21]. Dicer also contributes to the loading of mature miRNAs into the RNA-induced silencing complex (RISC). RISC is responsible for the gene silencing observed due to miRNA expression and RNA interference [22,23]. After loading into the RISC complex, miRNAs guide the RISC complex to their target genes by binding to imperfect complementary sites within the $3^{\prime}$ untranslated regions (3'UTRs) [24]. Studies have shown that miRNA can also bind to the $5^{\prime}$ untranslated regions (5'UTRs) and even the open reading frames (ORFs) of their target mRNAs [25]. miRNAs repress the expression of their target genes by mRNA destabilization and/or translational repression [24,26]. Moreover, miRNAs were also reported to cause histone modification and/or DNA methylation of promoter sites, which affects 
the expression of target genes [27,28].

\section{1 miRNAs in cardiac development}

Global disruption of the expression of all miRNAs in the heart is the first step to understand the function of miRNAs in cardiac development and physiology. Dicer, an RNase III endoribonuclease is a critical enzyme for the maturation of most miRNAs. Conventional deletion of Dicer caused early embryonic lethality in mice [29] and zebrafish [30,31], prior to the development of the heart. In order to study the function of miRNAs in cardiac development, studies using cardiac-specific promoter-driven Cre line, such as Nkx2.5-Cre and $\alpha$-MHC-Cre, to knockout Dicer in cardiac linage have been performed. Disrupting miRNA expression in early embryonic stage using $\mathrm{Nkx} 2.5$-Cre leads to improperly compacted ventricular myocardium in mutant embryos [32]. While $\alpha$-MHC-Cre-mediated conditional deletion of Dicer causes postnatal lethality due to dilated cardiomyopathy and heart failure [33]. Additionally, tamoxifen-induced cardiacspecific deletion of Dicer in adult hearts induces spontaneously cardiac hypertrophy and fetal gene expression [34]. Moreover, cardiac-specific knockout of Drosha leads to similar cardiac defects with that of cardiac-specific Dicer mutant, further supporting the importance of miRNAs in cardiac development and function [35].

Studies of miRNA expression profiling have indicated that although many miRNAs are expressed ubiquitously throughout the mammalian organisms, some miRNAs have tissue-specific expression patterns [36]. Focusing on striated muscles like cardiomyocytes and skeletal muscle cells, previous studies found that miR-1/133 cluster is the most abundantly expressed miRNA in the heart whose expression was detected as early as embryonic day 13.5 (E13.5) [37]. The expression of this miRNA cluster can be regulated by multiple myogenic transcription factors, including MyoD [38], Mef2 [39], and SRF [40]. miR-1, miR-133, together with miR-206 [41], miR-208a/b [42,43] and miR-499 [44] have been identified specifically expressed in striated muscles (and therefore referred to as "myomiRs"). Gain- and loss-of-function studies demonstrated that myomiRs play key roles in cardiomyocyte proliferation, cardiac morphogenesis and stress responsive cardiac remodeling [40,42,43]. Interestingly, recent reports showed that miRNAs, which are not restricted to striated muscles, are also required for the normal cardiogenesis. miR-138 is required for the elongation of ventricular cardiomyocytes by repressing the expression of cspg2 and notch $1 \mathrm{~b}$, which are normally restricted to the atrioventricular canal region, in the ventricle in zebrafish [45]. Loss-of-function of miR-218 in zebrafish affects the endocardial migration, and therefore affects the heart tube formation by regulating the Vegf singling [46].

\section{2 miRNAs mediate cardiac regeneration}

Mammalian adult cardiomyocytes are terminally differentiated cells with very limited regenerative ability. In response to injury and cell loss, adult mammalian hearts are unable to fully regenerate. However, recent evidences indicate that human cardiomyocytes are able to proliferate during the postnatal life $[47,48]$. Intriguingly, it was discovered that in rodent models hearts before postnatal day 7 have a good regenerative ability. Young hearts can regenerate the cardiac tissue without the formation of scar (fibrosis) after resection of the ventricle apex. However, the cardiac regenerative ability is lost by $7 \mathrm{~d}$ of age due to a significant decrease of the proliferative rate of cardiomyocytes [49].

Both protein-coding genes and non-coding RNA genes have been suggested to participate in the regulation of cardiac regeneration. For protein-coding genes, inhibition of p38 MAP kinase was found to induce mitosis in adult mammalian cardiomyocytes [50]. Treatment with FGF1 and p38 MAP kinase inhibitors regenerates the heart, reduces scarring, and improves cardiac function in rats with cardiac injury [51]. Non-coding RNAs, especially miRNAs, have also been found playing an important role in cardiac regeneration. miRNA profiling analyses indicated that the expression of miRNAs is regulated during cell cycle exit of cardiomyocyte shortly after born. Through profiling and comparing miRNA expression between postnatal day 1 and day 10 rat cardiomyocytes, Porrello et al. [52] identified members of the miR-15 family, including miR-195, miR-15a, miR-15b, miR-16 and miR-497, as important regulators of postnatal cardiomyocyte mitotic arrest. Further gain- and loss-of-function studies demonstrated that cardiomyocytes proliferation can be inhibited by this family of miRNAs through the repression of multiple cell cycle regulators, including the checkpoint kinase 1 (Chek1). Interestingly, miR-15 family was also shown up-regulated in cardiac ischemia and heart failure [53] and it was reported that miR-15 induces apoptosis by targeting anti-apoptotic factor Bcl2 [54]. Together, these studies suggest that the miR-15 family may play distinct roles in cardiomyocytes at different developmental and/or pathological conditions.

To further investigate if miRNAs can increase cardiomyocytes proliferation, Eulalio et al. [55] performed a highcontent, fluorescence-microscopy-based, high-throughput screening in neonatal rat cardiomyocytes using a wholegenome miRNA library. They identified about 40 miRNAs that strongly increased both DNA synthesis and cytokinesis in neonatal mouse and rat cardiomyocytes [55]. Two of these miRNAs, miR-590 and miR-199a, were further demonstrated to induce cardiomyocyte proliferation both in vitro and in vivo. Most recently, our group also discovered that other miRNAs can increase cardiomyocytes proliferation. Using both transgenic (TG) and knockout (KO) mice model, we demonstrated that miR-17-92 cluster is required 
for and sufficient to induce cardiomyocyte proliferation. More specifically, we identified miR-19a/b as the major contributors among the miR-17-92 cluster to the regulation of the cardiomyocyte proliferation. We further identified the tumor suppressor PTEN as a direct target of miR-17-92 repressed. These studies uncovered that miR-17-92 plays a key role in the regulation of cardiomyocytes proliferation in embryonic, postnatal and adult hearts [56].

Over the past several years, the approaches to differentiating embryonic stem cells and induced pluripotent stem cells (iPSCs) into cardiomyocytes to compensate the loss of cardiomyocytes during cardiac injury have raised the hope for patients with cardiac infarction and heart failure [57,58]. However, the potential tumorigenicity is a big concern for these strategies of cardiac regeneration [59,60]. Most recently, an exciting breakthrough was achieved in which Ieda et al. [61] found a new strategy to direct reprogram fibroblast $(\mathrm{CF})$ into cardiomyocyte (CM) through the combination of three cardiac-specific transcriptional factors, Gata4, Mef2c and Tbx5 (GMT) in vitro. Intriguingly, Qian et al. [62] and Song et al. [63] demonstrated that the direct reprogramming was also achievable in vivo. These investigators reported that they were able to use three factors (GMT) or four cardiac transcription factors, GATA4, HAND2, MEF2C, and TBX5 (GHMT) respectively, to reprogram cardiac fibroblasts or even mouse tail-tip fibroblasts into beating cardiomyocyte-like cells in vivo. More importantly, reprogramming cardiac fibroblasts into cardiomyocytes in vivo was shown to improve cardiac function and reduce cardiac fibrosis in a mouse model of myocardial infraction. It is not known whether miRNAs are involved in the process of reprogramming which is induced by cardiac transcription factors.

Given their characteristics of tiny and mighty, it is not surprising that a recent report indicated that a combination of miR-1, miR-133, miR-208, and miR-499 was able to directly induce the cellular reprogramming of fibroblasts into cardiomyocyte-like cells in vitro [64]. The investigators demonstrated that miR-1 alone is sufficient to induce the fibroblast to cardiomyocyte reprogramming. However, this reprogramming efficiency was dramatically enhanced when miRNAs 133, 208 and 499 were added. Interestingly, the process of reprogramming was further enhanced about 10 -fold after JAK inhibitor I treatment. Moreover, administration of miRNAs into ischemic mouse myocardium resulted in direct conversion of cardiac fibroblasts to cardiomyocytes in situ. Recently, Nam et al. [65] used a combination of transcription factors and miRNAs to induce the direct reprogramming of fibroblasts into cardiomyocyte-like cells. They treated human fibroblasts with four transcriptional factors, GATA-4, Hand2, Tbx5 and Myocardin [66], together with two miRNAs, miR-1 and miR-133. Portion of the treated human fibroblasts were reprogrammed into cells with sarcomere-like structures, showing spontaneous contractility after 4-11 weeks in culture. Besides the pheno- typic changes, the investigators found that the transcriptome of reprogrammed cells also shifted toward cardiomyocytes. These data indicated miRNAs could function with cardiac transcriptional factors to synergistically control cardiac reprogramming.

Unlike adult skeletal muscle, which contains a large population of satellite cells to serve as stem cells to regenerate the lost muscle mass in response to injury, the adult heart only has a very limited population of stem cells or progenitor cells. Studies indicated that these cells can be differentiated into cardiomyocyte in vitro. Interestingly, miRNAs are shown to contribute to the differentiation of cardiac stem cells (CSCs) into mature, working cardiomyocytes. Hosoda et al. [67] identified that miR-499 promotes the differentiation of human CSCs (hCSCs) into mechanically integrated cardiomyocytes by repressing its targets, Sox6 and Rod1. More importantly, the miR-499-overexpressed hCSCs injected into the infracted mouse hearts could enhance cardiac differentiation and further contribute to a better improvement of the cardiac function of the injured hearts.

\section{3 miRNAs regulate cardiac remodeling}

Cardiac remodeling, which is defined as alteration in the structure (dimensions, mass, shape) of the heart, is one of the major responses of the heart to biomechanical stress and pathological stimuli. Among them, cardiac hypertrophy is anatomically defined as an increase in the thickness of the cardiac ventricular wall, owing to enlargement of myocyte size and/or increased fibrosis. Sustained cardiac hypertrophy often leads to end stage heart failure. To investigate the involvement of miRNAs in this process, genome-wide profiling of miRNA expression has been performed and dysregulated miRNAs were identified during cardiac remodeling [68,69]. For instance, miR-21, a miRNA upregulated during hypertrophy, was shown to promote cardiac fibroblast survival through enhancing the ERK-MAP kinase activity [70]. Interestingly, inhibition of miR-21 via an antagomir was shown to repress cardiac hypertrophy and fibrosis in vivo in response to stress, however, these results could not be verified through genetic deletion of miR-21 in mice [71], indicating that miR-21 may not be essential for the pathological remodeling of the heart. Another study showed that isoproterenol-induced cardiac hypertrophy could be repressed when miR-23a was knocked down. miR-23a represents another miRNA up-regulated during hypertrophy and the repressive effect of miR-23a in cardiac hypertrophy was suggested, at least in part, due to the repression of MuRF1, an anti-hypertrophic factor [72]. Moreover, cardiac-specific overexpression of miR-195, another miRNA induced in hypertrophic hearts, in transgenic mice model induces significantly cardiac hypertrophy and dilated cardiomyopathy with unknown mechanism [53]. 
Recently, we and others [73,74] demonstrated that miR-22, a miRNA enriched in cardiomyocytes but only mildly up-regulated during cardiac hypertrophy, significantly promotes cardiac hypertrophy in vitro. Consistently, cardiacspecific knockout of miR-22 in mice showed the repressed cardiac hypertrophy was accompanied with accelerated dilation [73]. Conversely, cardiac-specific overexpression of this miRNA induced spontaneous hypertrophy growth in the heart [75]. Additional studies showed that miR-22 represses a broad spectrum of target genes, including Sirt1, HDAC4, PPARa and Purb, a negative regulator of SRF [73,75].

Conversely, unlike those up-regulated miRNAs, the expression of miR-93, miR-181 and muscle-specific microRNAs miR-1 and miR-133a is down-regulated during cardiac hypertrophy $[53,76,77]$. The miR-133a is coded by two genomic loci, miR-133a-1 and miR-133a-2. Studies showed that inhibition of miR-133a leads to hypertrophy both in vitro and in vivo, probably through the derepression of RhoA, Cdc42, and NELFA/WHsc2 [76]. Genetic studies indicated that deletion of both miR-133a-1 and miR-133a-2, but not each single one alone, leads to phenotypically abnormal, even embryonic lethal in mice [78]. All these data suggest that miR-133a is not only necessary for cardiac development, but also required for normal cardiac functional maintenance in adult hearts. miR-208a, whose expression was not altered in cardiac hypertrophy, has been demonstrated to be involved in stress-dependent cardiac hypertrophy by targeting thyroid hormone receptor associated protein 1 (THRAP1). Both gain- and loss-of-function studies demonstrated that miR-208 is not only sufficient to induce cardiac hypertrophy but also required for the hypertrophic growth under stress $[42,43]$.

Cardiac fibrosis, which is defined as abnormal deposition of collagen by cardiac fibroblast, is often observed to replace the "drop-out" of cardiomyocytes during cardiac remodeling. Many genes have been reported to participate in the regulation of this process. It is not surprising that miRNAs were reported to regulate cardiac fibrosis in recent years. Connective tissue growth factor (CTGF) is a key molecule in the process of fibrosis and therefore seemingly serves as an attractive therapeutic target [79-81]. However, it was unknown how CTGF transcripts were regulated posttranscriptionally. Duisters et al. [82] showed that miR-133 and miR-30 were involved in myocardial matrix remodeling through regulating CTGF. Both miR-133 and miR-30 were found consistently down-regulated in several models of heart failure and pathological hypertrophy. Knockdown of these miRNAs resulted in a strong increase of CTGF levels. Conversely, overexpression of miR-133 and miR-30c repressed the production of collagens, which was accompanied with a decrease in CTGF expression levels. Moreover, the miR-29 family, which was predominantly expressed in cardiac fibroblasts [83], is significantly down-regulated in the fibrotic border zone of infracted hearts. Intriguingly, many of the miR-29 downstream target genes, such as
FBN1, COL1A1, COL1A2, ELN and COL3A1, are upregulated after myocardial infarction, suggesting that they may contribute to miR-29-mediated cardiac fibrosis. Taken together, these studies indicate that miRNAs are not only important for cardiovascular development, but also essential factors for cardiac remodeling.

\section{4 miRNAs regulate ischemia and conduction system}

Ischemia is an independent risk factor of cardiovascular events, which leads to myocardial infarction (MI) and ischemia-reperfusion (I/R) injury. Several miRNAs are reported to participate in the regulation of these pathologic processes, especially the cardiomyocyte apoptosis following MI and I/R injury. miR-92a, a member of miR-17-92 cluster involved in cardiomyocyte proliferation, also participated in the control of cardiomyocyte survival through targeting integrin subunit $\alpha 5$ and eNOS [84]. Inhibition of miR-92a by antagomir has improved cardiac function and deduced cardiomyocyte apoptosis after myocardial infraction in mice. miR-21 serves as an anti-apoptotic factor in MI animal models through targeting PDCD4 and repressing its expression [85]. Unlike miR-21, miR-1/206 acts as a pro-apoptotic factor by repressing the anti-apoptosis gene $I G F-1$ in infracted heart. Both IGF-1 loss-of-function and miR-1/206 gain-of- function can increase caspase-3-mediated apoptotic signaling pathway [86]. Additionally, miR-320 is downregulated after I/R injury. Gain- and loss-of-function studies demonstrated that miR-320 promotes cardiomyocyte apoptosis via maintaining the HSP20 levels [87].

The cardiac conduction system can be damaged following cardiac injury, such as cardiac ischemia or acute MI. Cellular necrosis in the lesion region is able to cause the dysfunction of the whole cardiac conduction system, including sinoatrial node, atrioventricular node and His-Purkinje system. Some of the defects include that the electrical signals could not be conducted smoothly through this conduction system, following a series of arrhythmia. miRNAs have been shown to participate in this process and the proper expression of miRNAs is critical for sustaining the normal function of cardiac conduction system. For instance, it has been reported that miR-1 and miR-133 target several ion channel and gap-junction associated genes, such as HCN2, HCN4, KCNJ2, ERG and GJA1 (Cx43) [88-90]. Overexpression of miR-1 in infracted myocardium can promote arrhythmogenesis, whereas arrhythmia could be alleviated through deleting endogenous miR-1 [90]. miR-208a, another cardiac-specific miRNA, has also been demonstrated to play an important role in arrhythmogenesis [42], especially in the process of atrial depolarization, by regulating the expression of Connexin-40 (GJA5). Therefore, studies have established the role of miRNAs in the development and maintenance of the cardiac conduction 
system as well as in the pathology of cardiac ischemia.

\section{Conclusion and perspectives}

miRNA research has rapidly emerged as an important area within the field of non-coding RNAs. These newly characterized "small" regulators have proven to play "big" roles in multiple levels of cellular biological behavior, from cell proliferation, differentiation and apoptosis, to the maintenance of stem cell self-renewal. From cardiac point of view, miRNAs have been found to participate in nearly all processes of cardiovascular biology, such as heart development, cardiomyocyte regeneration, cardiac remodeling, as well as stress induced injury and heart diseases. However, the identified miRNAs and miRNA-targets thus far are just the tip of the iceberg, and numerous questions concerning the relationship between miRNAs and cardiac function remain to be answered.

miRNAs could be utilized as bio-markers for the diagnosis of cardiovascular disease, given that the expression of many miRNAs is altered in varieties of cardiac biologic processes and disease conditions. The "circulating miRNAs" are stable in mammalian serum and plasma, which are much easier to acquire, raising the possibility that they could serve as biomarkers for heart disease diagnosis and prediction [91,92]. Recently, a clinical study has already made progress toward this possibility by showing the circulating miR-192 level is correlated with the development of ischemic heart failure after acute myocardial infarction in human patients [93]. The research for translational medicine of miRNAs has emerged rapidly in recent years. Though the therapeutic potential of miRNAs in cardiovascular disease remains debatable, much remarkable progress has been made. Cardiologists are now attempting to use miRNAs and/or their inhibitors to treat heart diseases. Along with that, many efficient techniques to manipulate miRNA levels in vivo have been developed. Among them, antagomirs, which knock down targeted miRNAs via sequestering them from the functional complex, were shown to be stable in the blood [94]. Conversely, miRNA mimic, a synthesized chemically modified double-stranded oligonucleotide, can achieve the gain-of-function of specific miRNA in vitro and in vivo [95,96]. Additionally, rAAV9 vector has been demonstrated of high affinity for myocardium, which means it can be used for delivering miRNA-related therapeutic molecules to the heart specifically through intravenous injection [97]. With these efficient strategies for gain- and loss-of-function of the molecular mechanism and therapeutic application of miRNAs in cardiovascular disease, much more fruitful work about the molecular mechanism and therapeutic application of miRNAs in cardiovascular disease will emerge. We are confident that the diagnosis and therapeutics based on miRNA will play an even more important role in the field of gene therapy.
We thank members of Wang's lab for advice and support. Work in Wang's lab was supported by the March of Dimes Foundation (FY11-426) and the National Institutes of Health (HL085635). Huang Zhan-Peng is a postdoctoral fellow and Wang Da-Zhi is an Established Investigator of the American Heart Association.

1 Guttman M, Rinn J L. Modular regulatory principles of large non-coding RNAs. Nature, 2012, 482: 339-346

2 Wilusz J E, Sunwoo H, Spector D L. Long noncoding RNAs: functional surprises from the RNA world. Genes Dev, 2009, 23: 1494-1504

3 Bartel D P. microRNAs: target recognition and regulatory functions. Cell, 2009, 136: 215-233

4 Chen K, Rajewsky N. The evolution of gene regulation by transcription factors and microRNAs. Nat Rev Genet, 2007, 8: 93-103

5 Lee R C, Feinbaum R L, Ambros V. The C. elegans heterochronic gene lin-4 encodes small RNAs with antisense complementarity to lin-14. Cell, 1993, 75: 843-854

6 Wightman B, Ha I, Ruvkun G. Posttranscriptional regulation of the heterochronic gene lin-14 by lin- 4 mediates temporal pattern formation in C. elegans. Cell, 1993, 75: 855-862

7 Reinhart B J, Weinstein E G, Rhoades M W, et al. microRNAs in plants. Gen Dev, 2002, 16: 1616-1626

8 Lagos-Quintana M, Rauhut R, Lendeckel W, et al. Identification of novel genes coding for small expressed RNAs. Science, 2001, 294 : 853-858

9 Lau N C, Lim L P, Weinstein E G, et al. An abundant class of tiny RNAs with probable regulatory roles in Caenorhabditis elegans. Science, 2001, 294: 858-862

10 Lee R C, Ambros V. An extensive class of small RNAs in Caenorhabditis elegans. Science, 2001, 294: 862-864

11 Tanzer A, Stadler P F. Molecular evolution of a microRNA cluster. J Mol Biol, 2004, 339: 327-335

12 Molnár A, Schwach F, Studholme D J, et al. miRNAs control gene expression in the single-cell alga Chlamydomonas reinhardtii. Nature, 2007, 447: 1126-1129

13 Kren B T, Wong P Y, Sarver A, et al. microRNAs identified in highly purified liver-derived mitochondria may play a role in apoptosis. RNA Biol, 2009, 6: 65-72

14 Lee C T, Risom T, Strauss W M. Evolutionary conservation of microRNA regulatory circuits: an examination of microRNA gene complexity and conserved microRNA-target interactions through metazoan phylogeny. DNA Cell Biol, 2007, 26: 209-218

15 He L, Hannon G J. microRNAs: small RNAs with a big role in gene regulation. Nature, 2004, 5: 522-531

16 Carmell M A, Hannon G J. RNase III enzymes and the initiation of gene silencing. Nat Struct Mol Biol, 2004, 11: 214-218

17 Tijsterman M, Plasterk R H. Dicers at RISC: the mechanism of RNAi. Cell, 2004, 117: 1-3

18 Denli A M, Tops B B, Plasterk R H, et al. Processing of primary micro-RNAs by the microprocessor complex. Nature, 2004, 432: 231-235

19 Murchison E P, Hannon G J. miRNAs on the move: miRNA biogenesis and the RNAi machinery. Curr Opin Cell Biol, 2004, 16: 223-229

20 Lee Y, Ahn C, Han J, et al. The nuclear RNase III Drosha initiates microRNA processing. Nature, 2003, 425: 415-419

21 Lee Y, Jeon K, Lee J T, et al. microRNA maturation: stepwise processing and subcellular localization. EMBO J, 2002, 21: 4663-4670

22 Liu Q, Rand T A, Kalidas S, et al. R2D2, a bridge between the initiation and effector steps of the Drosophila RNAi pathway. Science, 2003, 301: 1921-1925

23 Tomari Y, Matranga C, Haley B, et al. A protein sensor for siRNA asymmetry. Science, 2004, 306: 1377-1380

24 Bartel D P. microRNAs: genomics, biogenesis, mechanism, and function. Cell, 2004, 116: 281-297

25 Tay Y, Zhang J, Thomson A M, et al. microRNAs to Nanog, Oct4 and Sox 2 coding regions modulate embryonic stem cell differentia- 
tion. Nature, 2008, 455: 1124-1128

26 Wu L, Fan J, Belasco J G. microRNAs direct rapid deadenylation of mRNA. Proc Natl Acad Sci USA, 2006, 103: 4034-4039

27 Tan Y, Zhang B, Wu T, et al. Transcriptional inhibition of Hoxd4 expression by miRNA-10a in human breast cancer cells. BMC Mol Biol, 2009, 10: 12

28 Hawkins P G, Morris K V. RNA and transcriptional modulation of gene expression. Cell Cycle, 2008, 7: 602-607

29 Bernstein E, Kim S Y, Carmell M A, et al. Dicer is essential for mouse development. Nat Genet, 2003, 35: 215-217

30 Wienholds E, Koudijs M J, van Eeden F J, et al. The microRNA-producing enzyme Dicer1 is essential for zebrafish development. Nat Genet, 2003, 35: 217-218

31 Giraldez A J, Cinalli R M, Glasner M E, et al. microRNAs regulate brain morphogenesis in zebrafish. Science, 2005, 308: 833-838

32 Saxena A, Tabin C J. miRNA-processing enzyme Dicer is necessary for cardiac outflow tract alignment and chamber septation. Proc Natl Acad Sci USA, 2010, 107: 87-91

33 Chen J F, Murchison E P, Tang R, et al. Targeted deletion of Dicer in the heart leads to dilated cardiomyopathy and heart failure. Proc Natl Acad Sci USA, 2008, 105: 2111-2116

34 da Costa Martins P A, Bourajjaj M, Gladka M, et al. Conditional dicer gene deletion in the postnatal myocardium provokes spontaneous cardiac remodeling. Circulation, 2008, 118: 1567-1576

35 Rao P K, Toyama Y, Chiang H R, et al. Loss of cardiac microRNA-mediated regulation leads to dilated cardiomyopathy and heart failure. Circ Res, 2009, 105: 585-594

36 Lagos-Quintana M, Rauhut R, Yalcin A, et al. Identification of tissue-specific microRNAs from mouse. Curr Biol, 2002, 12: 735-739

37 Chen J F, Mandel E M, Thomson J M, et al. The role of microRNA-1 and microRNA-133 in skeletal muscle proliferation and differentiation. Nat Genet, 2006, 38: 228-233

38 Rao P K, Kumar R M, Farkhondeh M, et al. Myogenic factors that regulate expression of muscle-specific microRNAs. Proc Natl Acad Sci USA, 2006, 103: 8721-8726

39 Liu N, Williams A H, Kim Y, et al. An intragenic MEF2-dependent enhancer directs muscle-specific expression of microRNAs 1 and 133. Proc Natl Acad Sci USA, 2007, 104: 20844-20849

40 Zhao Y, Samal E, Srivastava D. Serum response factor regulates a muscle-specific microRNA that targets Hand2 during cardiogenesis. Nature, 2005, 436: 214-220

41 Williams A H, Valdez G, Moresi V, et al. microRNA-206 delays ALS progression and promotes regeneration of neuromuscular synapses in mice. Science, 2009, 326: 1549-1554

42 Callis T E, Pandya K, Seok H Y, et al. microRNA-208a is a regulator of cardiac hypertrophy and conduction in mice. J Clin Invest, 2009, 119: 2772-2786

43 van Rooij E, Sutherland L B, Qi X, et al. Control of stress-dependent cardiac growth and gene expression by a microRNA. Science, 2007, 316: 575-579

44 van Rooij E, Quiat D, Johnson B A, et al. A family of microRNAs encoded by myosin genes governs myosin expression and muscle performance. Dev Cell, 2009, 17: 662-673

45 Morton S U, Scherz P J, Cordes K R, et al. microRNA-138 modulates cardiac patterning during embryonic development. Proc Natl Acad Sci USA, 2008, 105: 17830-17835

46 Fish J E, Wythe J D, Xiao T, et al. A Slit/miR-218/Robo regulatory loop is required during heart tube formation in zebrafish. Development, 2011, 138: 1409-1419

47 Bergmann O, Bhardwaj R D, Bernard S, et al. Evidence for cardiomyocyte renewal in humans. Science, 2009, 324: 98-102

48 Mollova M, Bersell K, Walsh S, et al. Cardiomyocyte proliferation contributes to heart growth in young humans. Proc Natl Acad Sci USA, 2013, 110: 1446-1451

49 Porrello E R, Mahmoud A I, Simpson E, et al. Transient regenerative potential of the neonatal mouse heart. Science, 2011, 331: 1078-1080

50 Engel1 F B, Schebesta M, Duong M T, et al. p38 MAP kinase inhibition enables proliferation of adult mammalian cardiomyocytes. Genes Dev, 2005, 19: 1175-1187
51 Engel F B, Hsieh P C, Lee R T, et al. FGF1/p38 MAP kinase inhibitor therapy induces cardiomyocyte mitosis, reduces scarring, and rescues function after myocardial infarction. Proc Natl Acad Sci USA, 2006, 103: 15546-15551

52 Porrello E R, Johnson B A, Aurora A B, et al. miR-15 family regulates postnatal mitotic arrest of cardiomyocytes. Circ Res, 2011, 109: 670-679

53 van Rooij E, Sutherland L B, Liu N, et al. A signature pattern of stress-responsive microRNAs that can evoke cardiac hypertrophy and heart failure. Proc Natl Acad Sci USA, 2006, 103: 18255-18260

54 Cimmino A, Calin G A, Fabbri M, et al. miR-15 and miR-16 induce apoptosis by targeting BCL2. Proc Natl Acad Sci USA, 2005, 102: 13944-13949

55 Eulalio A, Mano M, Dal Ferro M, et al. Functional screening identifies miRNAs inducing cardiac regeneration. Nature, 2012, 492: 376-381

56 Chen J, Huang Z P, Seok H Y, et al. mir-17-92 cluster is required for and sufficient to induce cardiomyocyte proliferation in postnatal and adult hearts. Circ Res, 2013, 112: 1557-1566

57 Takahashi K, Tanabe K, Ohnuki M, et al. Induction of pluripotent stem cells from adult human fibroblasts by defined factors. Cell, 2007, 131: 861-872

58 Takahashi K, Yamanaka S. Induction of pluripotent stem cells from mouse embryonic and adult fibroblast cultures by defined factors. Cell, 2006, 126: 663-676

59 Mummery C. Induced pluripotent stem cells: a cautionary note. N Engl J Med, 2011, 364: 2160-2162

60 Yamashita T, Kawai H, Tian F, et al. Tumorigenic development of induced pluripotent stem cells in ischemic mouse brain. Cell Transplant, 2011, 20: 883-891

61 Ieda M, Fu J D, Delgado-Olguin P, et al. Direct reprogramming of fibroblasts into functional cardiomyocytes by defined factors. Cell, 2010, 142: 375-386

62 Qian L, Huang Y, Spencer C I, et al. In vivo reprogramming of murine cardiac fibroblasts into induced cardiomyocytes. Nature, 2012, 485: 593-598

63 Song K, Nam Y J, Luo X, et al. Heart repair by reprogramming non-myocytes with cardiac transcription factors. Nature, 2012, 485: 599-604

64 Jayawardena $\mathrm{T}$ M, Egemnazarov B, Finch E A, et al. microRNA-mediated in vitro and in vivo direct reprogramming of cardiac fibroblasts to cardiomyocytes. Circ Res, 2012, 110: 1465-1473

65 Nam Y J, Song K, Luo X, et al. Reprogramming of human fibroblasts toward a cardiac fate. Proc Natl Acad Sci USA, 2013, 110: 5588-5593

66 Wang D, Chang P S, Wang Z, et al. Activation of cardiac gene expression by myocardin, a transcriptional cofactor for serum response factor. Cell, 2001, 105: 851-862

67 Hosoda T, Zheng H, Cabral-da-Silva M, et al. Human cardiac stem cell differentiation is regulated by a mircrine mechanism. Circulation, 2011, 123: 1287-1296

68 Tatsuguchi M, Seok H Y, Callis T E, et al. Expression of microRNAs is dynamically regulated during cardiomyocyte hypertrophy. J Mol Cell Cardiol, 2007, 42: 1137-1141

69 Callis T E, Wang D Z. Taking microRNAs to heart. Trends Mol Med, 2008, 14: 254-260

70 Thum T, Gross C, Fiedler J, et al. microRNA-21 contributes to myocardial disease by stimulating MAP kinase signalling in fibroblasts. Nature, 2008, 456: 980-984

71 Patrick D M, Montgomery R L, Qi X, et al. Stress dependent cardiac remodeling occurs in the absence of microRNA-21 in mice. J Clin Invest, 2010, 120: 3912-3916

72 Lin Z, Murtaza I, Wang K, et al. miR-23a functions downstream of NFATc3 to regulate cardiac hypertrophy. Proc Natl Acad Sci USA, 2009, 106: 12103-12108

73 Huang Z P, Chen J, Seok H Y, et al. MicroRNA-22 regulates cardiac hypertrophy and remodeling in response to stress. Circ Res, 2013, 112: $1234-1243$

74 Jentzsch C, Leierseder S, Loyer X, et al. A phenotypic screen to 
identify hypertrophy-modulating microRNAs in primary cardiomyocytes. J Mol Cell Cardiol, 2012, 52: 13-20

75 Gurha P, Abreu-Goodger C, Wang T, et al. Targeted deletion of microRNA-22 promotes stress-induced cardiac dilation and contractile dysfunction. Circulation, 2012, 125: 2751-2761

76 Care A, Catalucci D, Felicetti F, et al. microRNA-133 controls cardiac hypertrophy. Nat Med, 2007, 13: 613-618

77 Sayed D, Hong C, Chen I Y, et al. MicroRNAs play an essential role in the development of cardiac hypertrophy. Circ Res, 2007, 100: 416-424

78 Liu N, Bezprozvannaya S, Williams A H, et al. microRNA-133a regulates cardiomyocyte proliferation and suppresses smooth muscle gene expression in the heart. Genes Dev, 2008, 22: 3242-3254

79 Shi-Wen X, Leask A, Abraham D. Regulation and function of connective tissue growth factor/CCN2 in tissue repair, scarring and fibrosis. Cytokine Growth Factor Rev, 2008, 19: 133-144

80 Moussad E E, Brigstock D R. Connective tissue growth factor: what's in a name? Mol Gen Metab, 2000, 71: 276-292

81 Perbal B. CCN proteins: multifunctional signalling regulators. Lancet, 2004, 363: 62-64

82 Duisters R F, Tijsen A J, Schroen B, et al. miR-133 and miR-30 regulate connective tissue growth factor: implications for a role of microRNAs in myocardial matrix remodeling. Circ Res, 2009, 104: 170-178

83 van Rooij E, Sutherland L B, Thatcher J E, et al. Dysregulation of microRNAs after myocardial infarction reveals a role of miR-29 in cardiac fibrosis. Proc Natl Acad Sci USA, 2008, 105: 13027-13032

84 Bonauer A, Carmona G, Iwasaki M, et al. microRNA-92a controls angiogenesis and functional recovery of ischemic tissues in mice. Science, 2009, 324: 1710-1713

85 Dong $\mathrm{S}$, Cheng $\mathrm{Y}$, Yang J, et al. microRNA expression signature and the role of microRNA- 21 in the early phase of acute myocardial infarction. J Biol Chem, 2009, 284: 29514-29525

86 Shan Z X, Lin Q X, Fu Y H, et al. Upregulated expression of miR-1/
miR-206 in a rat model of myocardial infarction. Biochem Biophys Res Commun, 2009, 381: 597-601

87 Ren X P, Wu J, Wang X, et al. microRNA-320 is involved in the regulation of cardiac ischemia/reperfusion injury by targeting heat-shock protein 20. Circulation, 2009, 119: 2357-2366

88 Luo X, Lin H, Pan Z, et al. Down-regulation of miR-1/miR-133 contributes to re-expression of pacemaker channel genes $\mathrm{HCN} 2$ and HCN4 in hypertrophic heart. J Biol Chem, 2008, 283: 20045-20052

89 Xiao J, Luo X, Lin $\mathrm{H}$, et al. microRNA miR-133 represses HERG $\mathrm{K}^{+}$ channel expression contributing to QT prolongation in diabetic hearts. J Biol Chem, 2007, 282: 12363-12367

90 Yang B, Lin H, Xiao J, et al. The muscle-specific microRNA miR-1 regulates cardiac arrhythmogenic potential by targeting GJA1 and KCNJ2. Nature Med, 2003, 13: 486-491

91 Chen X, Ba Y, Ma L, et al. Characterization of microRNAs in serum: a novel class of biomarkers for diagnosis of cancer and other disease. Cell Res, 2008, 18: 997-1006

92 Mitchell P S, Parkin R K, Kroh E M, et al. Circulating microRNAs as stable blood-based markers for cancer detection. Proc Natl Acad Sci USA, 2008, 105: 10513-10518

93 Matsumoto S, Sakata Y, Nakatani D, et al. Circulating p53-responsive microRNAs are predictive indicators of heart failure after acute myocardial infarction. Circ Res, 2013, doi: 10.1161/ CIRCRESAHA.113.301209

94 Krutzfeldt J, Rajewsky N, Braich R, et al. Silencing of microRNAs in vivo with 'antagomirs'. Nature, 2005, 438: 685-689

95 Sehm T, Sachse C, Frenzel C, et al. miR-196 is an essential early-stage regulator of tail regeneration, upstream of key spinal cord patterning events. Dev Biol, 2009, 334: 468-480

96 Zeng L, Carter A D, Childs S J. miR-145 directs intestinal maturation in zebrafish. Proc Natl Acad Sci USA, 2009, 106: 17793-17798

97 Suckau L, Fechner H, Chemaly E, et al. Long-term cardiac-targeted RNA interference for the treatment of heart failure restores cardiac function and reduces pathological hypertrophy. Circulation, 2009, 119: $1241-1252$

Open Access This article is distributed under the terms of the Creative Commons Attribution License which permits any use, distribution, and reproduction in any medium, provided the original author(s) and source are credited. 